\title{
THE SYMPLECTIC STRUCTURE OF KÄHLER MANIFOLDS OF NONPOSITIVE CURVATURE
}

\author{
DUSA McDUFF
}

\begin{abstract}
In this note we show that the Kähler form on a simply connected complete Kähler manifold $W$ of nonpositive curvature is diffeomorphic to the standard symplectic form on $\mathbf{R}^{n}$. This means in particular that the symplectic structure on a Hermitian symmetric space of noncompact type is standard. We also show that if $L$ is a totally geodesic proper, connected Lagrangian submanifold of a complete Kähler manifold $W$ of nonpositive curvature, then $W$ is symplectomorphic to the cotangent bundle $T^{*} L$ with its usual symplectic structure provided that the fundamental group $\pi_{1}(W, L)$ vanishes. The proofs use a comparison theorem due to Greene \& Wu and Siu \& Yau.
\end{abstract}

\section{Introduction}

Let $W$ be a Kähler manifold with a pole, i.e., a point $p$ at which the exponential map is a diffeomorphism from the tangent space $W_{p}$ onto $W$. Following [2], we will call a 2-dimensional subspace of the tangent space $W_{x}$ a radial plane, if either $x=p$ or the subspace contains the tangent to the unique geodesic from $p$ through $x$. The radial curvature of $(W, p)$ is then the restriction of the sectional curvature function to the radial planes. Our first result is

Theorem 1. Let $(W, p)$ be a Kähler manifold with a pole such that the radial curvature is nonpositive. Then there is a diffeomorphism from $W$ to $\mathbf{R}^{n}$ which takes the Kähler form $\omega$ on $W$ to the standard symplectic form on $\mathbf{R}^{n}$.

Note that any simply connected, complete Kähler manifold with nonpositive curvature satisfies the hypotheses. There are many such manifolds (see [1] for example). Observe also that the symplectomorphism which we construct from $W$ to $\mathbf{R}^{n}$ is not in general holomorphic, for if it were it would preserve the Kähler metric.

Now suppose that $L$ is a totally geodesic, connected and properly embedded Lagrangian submanifold of a complete Kähler manifold $(W, \omega)$ of nonpositive

Received April 23, 1987 and, in revised form, October 26, 1987. The author was partially supported by National Science Foundation grant DSM-8504355. 
sectional curvature. If $\pi_{1}(W, L)=0$, the exponential map induces an isomorphism from the normal bundle $\nu_{L}$ onto $W$. (This may be seen by passing to the universal covers $\widetilde{L} \rightarrow \widetilde{W}$.) Thus, identifying $T^{*} L$ with $\nu_{L}$ via the symplectic form $\omega$, we get a diffeomorphism $\psi: T^{*} L \rightarrow W$. Let $\omega_{\text {can }}$ denote the canonical symplectic form on $T^{*} L$.

Theorem 2. Under the conditions, $\psi$ is isotopic to a symplectomorphism $\left(T^{*} L, \omega_{\text {can }}\right) \rightarrow(W, \omega)$.

Corollary. If $(V, \omega)$ is a compact Kähler manifold of nonpositive curvature with fundamental group $\Gamma$, then its cotangent bundle $\left(T^{*} V, \omega_{\text {can }}\right)$ is symplectomorphic to $(\widetilde{V} \times \widetilde{V} / \Gamma, \omega \oplus-\omega)$, where $\tilde{V}$ is the universal cover of $V$ and $\Gamma$ acts diagonally.

Proof of Corollary. Apply Theorem 2 to the image $L$ of the diagonal in $\tilde{V} \times \tilde{V} / \Gamma$. q.e.d.

As noted by Sikorav [6], this corollary allows one to establish Arnold's fixed point conjecture for $(V, \omega)$.

Theorems 1 and 2 are proved by replacing the given Kähler forms by other equivalent forms for which the results are easy to prove. These new Kähler forms arise from the Levi forms of certain plurisubharmonic functions: for Theorem 1 we consider the function whose value at $x$ is the distance of $x$ from the pole $p$, and for Theorem 2 we consider the distance of $x$ from $L$. The key step in the proofs is a comparison theorem which estimates the Levi forms of these functions in terms of the original Kähler metrics.

We will prove Theorem 1 in $\S 2$, and Theorem 2 in $\S 3$. I wish to thank the many mathematicians with whom I have had useful discussions about these results, and, in particular, J. D'Atri, C. Fefferman, F. Laudenbach, C. LeBrun, J.-C. Sikorav, R. Spatzier, and A. Weinstein.

\section{Proof of Theorem 1}

We begin by stating the comparison theorem which we will use. Given a real-valued function $f$ on $W, L f$ will denote its Levi form. For convenience, we will use "real" rather than "complex" notation, and so will define $L f$ to be the symmetric form on the tangent bundle $T W$ of $W$ given by

$$
L f(X, Y)=-d J d f(X, J Y),
$$

where $J$ is the canonical almost complex structure on $T W$. Note that the symmetry of $L f$ is equivalent to the identity

$$
d J d f(X, Y)=d J d f(J X, J Y)
$$

for the corresponding 2-form. 
Lemma 2.1 [2, Proposition 2.24]. Let $(W, p)$ be a Kähler manifold with a pole, $G$ the Kähler metric on $W$, and $r(x)$, the distance of $x$ from $p$. If the radial curvature of $W$ is nonpositive, then $L\left(r^{2}\right) \geq 4 G$.

Thus, $L\left(r^{2}\right)$ is a Kähler metric on $W$. Let $\omega_{r}=-d J d\left(r^{2}\right)$ be the corresponding Kähler form. We first show

Proposition 2.2. $\left(W, \omega_{r}\right)$ is symplectomorphic to $\mathbf{R}^{2 n}$ with its standard symplectic form $\omega_{0}$.

Next we use Moser's stability theorem [4] to prove

Proposition 2.3. $(W, \omega)$ is symplectomorphic to $\left(W, \omega_{r}\right)$.

Clearly, these two propositions constitute a proof of Theorem 1.

Proof of Proposition 2.2. Let $\|\cdot\|_{0}$ be the Kähler norm on $T W$ and $\|\cdot\|_{r}$ the norm induced by $L\left(r^{2}\right)$. Then Lemma 2.1 implies that $\|X\|_{r} \geq 4\|X\|_{0}$ for all tangent vectors $X$. Correspondingly, $4\|\eta\|_{r} \leq\|\eta\|_{0}$ on cotangent vectors $\eta$.

Consider the vector field $\xi$ given by

$$
\xi\lrcorner \omega_{r}=-J d\left(r^{2}\right) .
$$

Then $\left.d\left(r^{2}\right)(X)=J(\xi\lrcorner \omega_{r}\right)(X)=\omega_{r}(\xi, J X)=\langle\xi, X\rangle_{r}$. Thus $\xi$ is just the gradient of $r^{2}$ with respect to the inner product $\langle,\rangle_{r}$, and $\xi$ is everywhere transverse to the spheres $r=$ const. Further,

$$
\begin{aligned}
\|\xi\|_{0} \leq\|\xi\|_{r} & =\left\|J d\left(r^{2}\right)\right\|_{r} \quad \text { by }(*) \\
& \leq\left\|J d\left(r^{2}\right)\right\|_{0}=2 r\|d r\|_{0}=2 r .
\end{aligned}
$$

Therefore, because $W$ is complete with respect to $\|\cdot\|_{0}, \xi$ integrates up to a family $g_{t}$ of diffeomorphisms of $W$.

Now consider the radial vector field $\xi_{0}=\frac{1}{2} \Sigma_{i} x^{i} \partial / \partial x^{i}$ on $\mathbf{R}^{2 n}$. Observe that $\left.d\left(\xi_{0}\right\lrcorner \omega_{0}\right)=\omega_{0}$. We claim that when $\xi$ is expressed with respect to suitable coordinates, its 1 -jet at $p$ agrees with that of $\xi_{0}$ at 0 . In fact, if the complex coordinates $\left(x^{1}+i x^{2}, \cdots, x^{2 n-1}+i x^{2 n}\right)$ are normal at $p$ for the original Kähler metric $G$ on $W$, then $G=\Sigma G_{j k} d x^{j} d x^{k}$, where the 1-jet of $\left\{G_{j k}\right\}$ at $p$ equals $\left\{\delta_{j k}\right\}$. Hence, if $r_{0}$ denotes the Euclidean distance function $r_{0}(x)=\left(\Sigma\left(x^{i}\right)^{2}\right)^{1 / 2}$, then

$$
r(x)=r_{0}(x)+O\left(r_{0}\left(x^{3}\right)\right) .
$$

An easy calculation now shows that the 1-jet of $\xi$ is claimed.

Therefore, by [7], $\xi$ is conjugate to $\xi_{0}$ near $p$. In view of the nature of the flows of $\xi$ and $\xi_{0}$, this local conjugacy may be extended to a diffeomorphism from $W$ to $\mathbf{R}^{2 n}$ which pushes $\xi$ forward to $\xi_{0}$. Thus $\omega_{r}$ is pushed forward to a form $\omega^{\prime}$ such that $\left.\xi_{0}\right\lrcorner \omega^{\prime}=\omega^{\prime}$, and it remains to observe 
Lemma 2.4. Any symplectic form $\omega^{\prime}$ on $\mathbf{R}^{2 n}$ such that $\left.d\left(\xi_{0}\right\lrcorner \omega^{\prime}\right)=\omega^{\prime}$, is diffeomorphic to $\omega_{0}$.

Proof. Because $\left.\mathscr{L}_{\xi_{0}}\left(\omega^{\prime}\right)=d\left(\xi_{0}\right\lrcorner \omega^{\prime}\right)=\omega^{\prime}$, the flow $g_{t}$ of $\xi_{0}$ is such that $g_{t}^{*}\left(\omega^{\prime}\right)=e^{t} \omega^{\prime}$. But $g_{t}$ is just the multiplication by $e^{t / 2}$. Therefore, if $\omega^{\prime}=$ $\Sigma a_{j k} d x^{j} \wedge d x^{k}$, the functions $a_{j k}$ are constant along the rays through 0 . Since $\omega^{\prime}$ is continuous at 0 , the $a_{j k}$ are constant. Thus $\omega^{\prime}$ is linear, and the desired result follows. q.e.d.

This completes the proof of Proposition 2.2.

Proof of Proposition 2.3. Let $\tau_{t}=(1-t) \omega+t \omega_{r}, 0 \leq t \leq 1$. Then $\tau_{t}$ is a symplectic form for each $t$, and by Lemma 2.1 the norm $\|\cdot\|_{t}$ given by $\|X\|_{t}^{2}=\tau_{t}(X, J X)$ satisfies

$$
\|X\|_{t} \geq\|X\|_{0} \quad \text { for all } t .
$$

We want to find a family $g_{t}$ of diffeomorphisms of $W$ such that $g_{t}^{*}\left(\tau_{t}\right)=\omega$. According to [4], this may be done by letting $g_{t}$ be the flow of a family of vector fields $X_{t}$, and observing that the equation

$$
\left.0=\frac{d}{d t}\left(g_{t}^{*} \tau_{t}\right)=g_{t}^{*}\left(\mathscr{L}_{X_{t}}\right\lrcorner \tau_{t}+\dot{\tau}_{t}\right)
$$

is satisfied if $\left.X_{t}\right\lrcorner \tau_{t}+\lambda_{t}=0$, where $\lambda_{t}$ is a 1-form such that $\dot{\tau}_{t}=\frac{d}{d t}\left(\tau_{t}\right)=d \lambda_{t}$. To make the method work, one must check that the flow of $X_{t}$ is complete, i.e., that the $g_{t}$ are indeed diffeomorphisms.

In our case, we may take $\lambda_{t}=-J d\left(r^{2}\right)+\lambda$, where $\lambda$ is some solution of $d \lambda=\omega$. The latter equation can be solved by integrating $\omega$ along the geodesic rays from the pole $p$. It follows from Rauch's comparison theorem that the resulting 1-form $\lambda$ satisfies $\|\lambda\|_{0}=O(r)$. (Details of this calculation are given in [3].) Hence

$$
\left\|X_{t}\right\|_{0} \leq\left\|X_{t}\right\|_{t}=\left\|\lambda_{t}\right\|_{t} \leq\left\|\lambda_{t}\right\|_{0} \leq\left\|J d\left(r^{2}\right)\right\|_{0}+\|\lambda\|_{0}=O(r) .
$$

Because $W$ is complete with respect to $\|\cdot\|_{0}$, this implies that the $g_{t}$ are diffeomorphisms, as required.

\section{Proof of Theorem 2}

We will begin the proof of Theorem 2 by establishing the necessary comparison theorem. In the situation of Theorem 2, define $\rho(x)$, for $x \in W$, to be the distance from $x$ to $L$, and let $G_{\rho}$ be the Levi form of the function $\rho^{2}$. We write $G$ for the original Kähler metric on $W$.

Lemma 3.1. There is a constant $\varepsilon>0$ such that $G_{\rho} \geq \varepsilon G$.

Proof. We argue essentially by comparing $G_{\rho}$ with the Levi form of the function $r^{2}$ on $\mathbf{C}^{n}$, where $r(z)$ is the distance of $z$ to $\mathbf{R}^{n} \subset \mathbf{C}^{n}$. We will use 
the notation of Greene \& Wu. In particular, the Hessian $D^{2} f$ of a function $f$ on $W$ is given by

$$
D^{2} f(X, Y)=X(Y f)-\left(\nabla_{X} Y\right) f
$$

where $\nabla$ is the Levi-Civita connection of $G$. Because $G$ is Kähler, the Levi form of $\rho^{2}$ is related to its Hessian by the formula

$$
G_{\rho}(X, X)=D^{2}\left(\rho^{2}\right)(X, X)+D^{2}\left(\rho^{2}\right)(J X, J X)
$$

(see [2, Lemma 1.13]). Note also that

$$
D^{2}\left(\rho^{2}\right)=2\left[d \rho \otimes d \rho+\rho D^{2} \rho\right] .
$$

Let $\partial$ denote the gradient vector field of $\rho$ with respect to the metric $G$. Since $\partial$ is not defined on $L$, we will restrict attention from now on to $W-L$. This is permissible since it clearly suffices to establish the given inequality on $W-L$. Observe that

$$
D^{2} \rho(\partial, \partial)=\partial(\partial \rho)-\left(\nabla_{\partial} \partial\right) \rho=\partial(1)=0,
$$

since $\nabla_{\partial} \partial=0$ because $\partial$ is tangent to the normal geodesics from $L$. Note also that if the vector field $X$ is perpendicular to $\partial$, then

$$
\begin{aligned}
D^{2} \rho(X, \partial) & =\partial(X \rho)-\left(\nabla_{\partial} X\right) \rho=-\left\langle\nabla_{\rho} X, \partial\right\rangle \quad \text { since } X \rho=0 \\
& =-\nabla_{\rho}\langle X, \partial\rangle+\left\langle X, \nabla_{\partial} \partial\right\rangle=0 .
\end{aligned}
$$

Hence, as in [2, proof of Theorem A], the splitting $T_{x} W=\operatorname{span} \partial \oplus \partial^{\perp}$, which is $G$-orthogonal by definition, is also orthogonal with respect to the quadratic form $D^{2} \rho$.

We now claim that

$$
D^{2} \rho(X, X) \geq 0 \text { for all tangent vectors } X \text { on } W-L .
$$

By (3) and (4) above, this will follow if we show that

$$
D^{2} \rho(X, X) \geq 0 \quad \text { for all } X \perp \partial .
$$

Consider a tangent vector $X$ which is perpendicular to $\partial$ at $x \in W-L$, and let $b=\rho(x)$. Then $X$ is tangent to the level surface $S(b)=\{y: \rho(y)=b\}$. Choose a path $\xi:[-a, a] \rightarrow S(b)$ which is tangent to $X$ at 0 , and let $\gamma_{s}(t)$, for $0 \leq t \leq b$ and $s$ near 0 , be a family of geodesics from $L$ to $S(b)$ which are parametrized by arc length and such that $\gamma_{s}(B)=\xi(s)$ for all $s$ (see the figure). (Note that these geodesics are all perpendicular to $L$ since the distance of $\xi(s)$ from $L$ is $b$.) Define the vector field $W_{X}$ along $\gamma_{0}$ by

$$
W_{X}(t)=\left.\frac{d}{d s}\left[\gamma_{s}(t)\right]\right|_{s=0}
$$




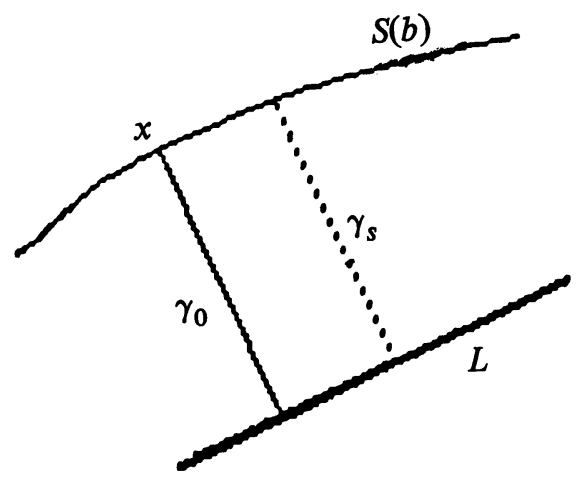

Then $W_{X}(b)=X$ by construction, and $Y=W_{X}(0)$ is tangent to $L$. Let denote $d / d t$, so that $\dot{\gamma}_{0}$ is the tangent vector along $\gamma_{0}$. We may extend $X$, and, correspondingly, $W_{X}$ and $Y$, to be vector fields. Then the second variation formula gives

$$
0=\left\langle\nabla_{X} X, \dot{\gamma}_{0}\right\rangle-\left\langle\nabla_{Y} Y, \dot{\gamma}_{0}\right\rangle+\int_{0}^{b}\left\langle\dot{W}_{X}, \dot{W}_{X}\right\rangle-\left\langle R\left(W, \dot{\gamma}_{0}\right) W, \dot{\gamma}_{0}\right\rangle d t
$$

Because $L$ is totally geodesic, $\nabla_{Y} Y$ is tangent to $L$, so that $\left\langle\nabla_{Y} Y, \dot{\gamma}_{0}\right\rangle=0$. Since the curvature term in $R$ is nonpositive, we have

$$
\left\langle\nabla_{X} X, \dot{\gamma}_{0}\right\rangle+\int_{0}^{b}\left\langle\dot{W}_{X}, \dot{W}_{X}\right\rangle d t \leq 0
$$

But

$$
\begin{aligned}
D^{2} \rho(X, X) & =X(X \rho)-\left(\nabla_{X} X\right) \rho=-\left\langle\nabla_{X} X, \partial\right\rangle \quad \text { since } X \rho=0 . \\
& =-\left\langle\nabla_{X} X, \dot{\gamma}_{0}\right\rangle \quad \text { since } \dot{\gamma}_{0}=\partial \text { along } \gamma_{0} .
\end{aligned}
$$

Thus $D^{2} \rho(X, X) \geq \int_{0}^{b}\left\|\dot{W}_{X}\right\|^{2} d t \geq 0$ as required.

Next we show that if $X$ is a unit vector at $x$, which is perpendicular to $\partial$ and such that $D^{2} \rho(X, X) \leq \varepsilon / b$, where $b=\rho(x)$, then

$$
\left\|W_{X}(0)\right\| \geq 1-\sqrt{\varepsilon} .
$$

(Note that in the model situation on $\mathrm{C}^{n}, D^{2} r(X, X)=0$ iff $X$ is totally real, and then $W_{X}(0)=X$.) To prove this claim, observe first that our hypotheses on $X$ imply that $\int_{0}^{b}\left\|\dot{W}_{X}\right\|^{2} d t \leq \varepsilon / b$. Let $U_{X}(t)$ be the parallel translate of $X$ along $\gamma_{0}$, and let $Y(t)=W_{X}(t)-U_{X}(t)$. Then $\dot{Y}(t)=\dot{W}_{X}(t), Y(b)=0$, and

$$
\left|\frac{d}{d t}\|Y(t)\|\right|=\frac{|\langle\dot{Y}, Y\rangle|}{\|Y\|} \leq\|\dot{Y}\|=\left\|\dot{W}_{X}\right\|
$$


Therefore,

$$
\|Y(0)\|=\left|\int_{0}^{b} \frac{d}{d t}\|Y\| d t\right| \leq \int_{0}^{b}\|\dot{Y}\| d t \leq\left(b \cdot \int_{0}^{b}\left\|\dot{W}_{X}\right\|^{2} d t\right)^{1 / 2} \leq \sqrt{\varepsilon} .
$$

Since $\left\|U_{X}(t)\right\|=1$ for all $t$, we have $\left\|W_{X}(0)\right\| \geq 1-\sqrt{\varepsilon}$, as claimed.

We complete the proof by showing that if $\mathscr{L}\|X\|=1$, then at least one of $D^{2} \rho^{2}(X, X)$ and $D^{2} \rho^{2}(J X, J X)$ is not less than $\varepsilon$. To do this, write $X$ as $\mu \partial+\lambda J \partial+\nu X^{\prime}$, where $X^{\prime}$ is perpendicular to both $\partial$ and $J \partial$ and has norm 1. If $\mu^{2} \geq \varepsilon / 2$, we find using (2), (4) and (6) that $D^{2} \rho^{2}(X, X) \geq$ $D^{2} \rho^{2}(\mu \partial, \mu \partial)=2 \mu^{2} \geq \varepsilon$. Similarly, $\lambda^{2} \geq \varepsilon / 2$ implies that $D^{2} \rho^{2}(J X, J X) \geq$ $\varepsilon$. Therefore, let us suppose that $\mu^{2}$ and $\lambda^{2}$ are both less than $\varepsilon / 2$. Note that $J X=-\lambda \partial+\mu J \partial+\nu X^{\prime \prime}$ where $X^{\prime \prime}=J X^{\prime}$, and let $\underline{X}^{\prime}=\lambda J \partial+\nu X^{\prime}$ and $\underline{X}^{\prime \prime}=\mu J \partial+\nu X^{\prime \prime}$. It suffices to show that at least one of $D^{2} \rho\left(\underline{X}^{\prime}, \underline{X}^{\prime}\right)$ and $D^{2} \rho\left(\underline{X}^{\prime \prime}, \underline{X}^{\prime \prime}\right)$ is not less than $\varepsilon / b$. We will suppose not, and derive a contradiction.

By (7), we know that

$$
\left\|W_{\underline{X}^{\prime}}(0)\right\| \text { and }\left\|W_{\underline{X}^{\prime \prime}}(0)\right\| \text { are both not less than } 1-\sqrt{\varepsilon} .
$$

By hypothesis on $\lambda$ and $\mu,\left\|J \underline{X}^{\prime}-\underline{X}^{\prime \prime}\right\|<\sqrt{\varepsilon}$. Because parallel translation commutes with $J$, it follows that $\left\|U_{\underline{X}^{\prime \prime}}(t)-J U_{\underline{X}^{\prime}}(t)\right\|<\sqrt{\varepsilon}$ for all $t$. Therefore

$$
\begin{aligned}
\left\|W_{\underline{X}^{\prime \prime}}(0)-J W_{\underline{X}^{\prime}}(0)\right\| \leq & \left\|W_{\underline{X}^{\prime \prime}}(0)-U_{\underline{X}^{\prime \prime}}(0)\right\|+\sqrt{\varepsilon} \\
& +\left\|J U_{\underline{X}^{\prime}}(0)-J W_{\underline{X}^{\prime}}(0)\right\| \\
\leq & 3 \sqrt{\varepsilon} \text { by the proof of }(7) .
\end{aligned}
$$

Since $W_{X}(0)$ is tangent to the Lagrangian submanifold $L$ for any $X$, and $J$ takes $T L$ to $T L^{\perp}$, the inequalities (8) and (9) are incompatible when $\varepsilon$ is sufficiently small. q.e.d.

With this lemma in hand, it is an easy matter to prove

Proposition 3.2. $(W, \omega)$ is symplectomorphic to $\left(W, \omega_{p}\right)$.

Proof. This follows by applying Moser's stability theorem as in Proposition 2.3. One must check that $\omega$ may be written as $d \lambda$ where $\lambda$ is a 1 -form such that $\|\lambda\|=0(\rho)$. It follows from Rauch's comparison theorem that a suitable $\lambda$ may be found by integrating $\omega$ along the normal geodesics from $L$. Further details are left to the reader. q.e.d.

It remains to show

Proposition 3.3. The forms $\psi^{*}\left(\omega_{\rho}\right)$ and $\omega_{\text {can }}$ on $T^{*} L$ are diffeomorphic.

As in the proof of Proposition 2.2, we establish this by considering the corresponding Liouville vector fields, and replacing Lemma 2.4 by Theorem 3.5 of [5]. Thus, let $\xi_{0}$ be the canonical vector field on $T^{*} L$, that is, the vector 
field which generates the action of $\mathbf{R}$ given by $\lambda \cdot X=e^{\lambda} X$, and let $\xi_{\rho}$ be the vector field on $W$ which is defined by the equation $\left.\xi_{\rho}\right\lrcorner \omega_{\rho}=-J d \rho^{2}$.

As a first step, we show

Lemma 3.4. The 1-jets of $\psi_{*} \xi_{0}$ and $\xi_{\rho}$ agree along $L$.

Proof. Let $z^{j}=x^{j}+i y^{j}, j=1, \cdots, n$, be (Kähler) normal coordinates about the point $p \in L$, chosen so that $T_{p} L=\bigcap_{j} \operatorname{ker} d y^{j}$. Then, if $G_{0}=$ $2 \sum\left(d x^{j}\right)^{2}+2 \sum\left(d y^{j}\right)^{2}$ is the flat metric, we know that

$$
J_{p}^{1}(G)=J_{p}^{1}\left(G_{0}\right)
$$

where $J_{p}^{k}$ denotes the $k$ th jet at $p$. Because the Christoffel symbols $\Gamma_{j k}^{i}$ depend on the 1-jet of the metric, the geodesic equation $\ddot{x}^{i}+\Gamma_{j k}^{i} \dot{x}^{j} \dot{x}^{k}=0$ implies that the exponential maps $T_{p} W \rightarrow W$ of $G_{0}$ and $G$ have the same 2-jet at $p$. Hence, being totally geodesic, $L$ is 2 -tangent at $p$ to the submanifold $L_{0}$ defined by the equations $y^{j}=0, j=1, \cdots, n$. Thus there is a local diffeomorphism $f$ of $W$, which takes $L$ to $L_{0}$ and is such that $J_{p}^{2}(f)=J_{p}^{2}(\mathrm{id})$. It follows easily that $\psi$ has the same 2-jet at $p$ as the composite

$$
T^{*} L \equiv \tau_{L} \stackrel{f^{*}}{\longrightarrow} \tau_{L_{0}} \stackrel{\exp _{G}}{\longrightarrow} W,
$$

and hence as the map $\psi_{0}: T^{*} L \rightarrow W$, which is given by the formula above, except with $G$ replaced by $G_{0}$. Since $f_{*} \xi_{0}$ is the canonical vector field of the bundle $\tau_{L_{0}}$, we have

$$
J_{p}^{1}\left(\psi_{*} \xi_{0}\right)=J_{p}^{1}\left(\psi_{0 *} \xi_{0}\right)=\sum y^{j} \partial / \partial y^{j}
$$

To calculate $J_{p}^{1}\left(\xi_{\rho}\right)$, define the function $\rho_{0}$ near $p$ by

$$
\rho_{0}(q)=G_{0} \text {-distance of } q \text { from } L_{0}=\left(\sum(y j)^{2}\right)^{1 / 2} \text { near } p \text {. }
$$

The recipe which gives us $\xi_{\rho}$ from $\rho$ yields the vector field $\sum y^{j} \partial / \partial y^{j}$ when applied to $\rho_{0}$. Since $J_{p}^{1}\left(\xi_{\rho}\right)$ depends on $J_{p}^{3}\left(\rho^{2}\right)$, we just have to show that $\rho^{2}$ and $\rho_{0}^{2}$ have the same 3-jet at $p$. This would follow if $\rho=\rho_{0}+O\left(\rho_{0}^{3}\right)$ near $p$. But

$$
\begin{aligned}
\rho(q) & =G \text {-distance of } q \text { from } L \\
& =f_{*} G \text {-distance of } f(q) \text { from } f(L)=L_{0},
\end{aligned}
$$

and, if we define $\rho^{\prime}$ by $\rho^{\prime}(q)=G_{0}$-distance of $f(q)$ from $L_{0}$, it is not hard to check that both $\rho-\rho^{\prime}$ and $\rho^{\prime}-\rho_{0}$ are $O\left(\rho_{0}^{3}\right)$. (For the first step, note that $J_{p}^{1}\left(f_{*} G\right)=J_{p}^{1}\left(G_{0}\right)$.)

Proof of Proposition 3.3. Just as with $\xi$ in Proposition 2.2, $\xi_{\rho}$ is the gradient of $\rho^{2}$ with respect to the metric $G_{\rho}$. Therefore $\xi_{\rho}$ is $G_{\rho}$-perpendicular to the level surfaces $\rho=$ const and integrates up to a complete flow. Lemma 
3.4 shows that its 1 -jet along its zero set $L$ agrees with that of the canonical homothety of some bundle over $L$. According to Theorem 3.5 of [5], under these conditions $W$ has a unique structure as a vector bundle over $L$ for which $\xi_{\rho}$ is the canonical homothety. Clearly, this vector bundle is isomorphic to $\tau_{L} \equiv T^{*} L$. Moreover, this isomorphism must take $\xi_{0}$ to $\xi_{\rho}$ and hence $\omega_{\text {can }}$ to $\omega_{\rho}$ (see Theorem 4.1 in [5]). The desired result follows.

\section{References}

[1] R. Greene, Function theory of noncompact Kähler manifolds of nonpositive curvature, Annals of Math. Studies, No. 102 (S. Yau, ed.), Princeton University Press, Princeton, NJ, 1982.

[2] R. Greene \& H. Wu, Function theory on manifolds which possess a pole, Lecture Notes in Math., Vol. 699, Springer, Berlin, 1979.

[3] D. McDuff, Symplectic structures on $\mathbf{R}^{2 n}$, in "Aspects dynamiques et topologiques des groupes infinis de transformation de la mechanique", Dazord, Desolneux-Moulis, (ed.), Travaux en Cours \#25, Hermann, Paris, 1987.

[4] J. Moser, On the volume elements on a manifold, Trans. Amer. Math. Soc. 120 (1965) 286-294.

[5] T. Nagano, 1-forms with their exterior derivative of maximal rank, J. Differential Geometry 2 (1968) 253-264.

[6] J.-C. Sikorav, Problèmes d'intersection et de points fixes en géométrie Hamiltonienne, Invent. Math., to appear.

[7] S. Sternberg, On contractions and a theorem of Poincaré, Amer. J. Math. 79 (1957) 809-824.

StATE UNIVERSITY OF NEW YORK, STONY BROOK 
